\title{
EL IMPACTO DEL CAMINO DE SANTIAGO A ESCALA LOCAL: LA PERCEPCIÓN DE LOS RESIDENTES DE DOS MUNICIPIOS GALLEGOS ${ }^{1}$
}

\author{
Melchor Fernández Fernández** \\ Universidade de Santiago de Compostela \\ https://orcid.org/0000-0002-6255-2033
}

Diana Fernández. Méndez**

Universidade de Santiago de Compostela https://orcid.org/0000-0003-0380-7232

Dolores Riveiro García**

Universidade de Santiago de Compostela

https://orcid.org/0000-0003-0104-4331

\section{RESUMEN}

Este trabajo estudia la percepción de los residentes de dos municipios rurales sobre los impactos económicos, sociales, culturales y medioambientales del Camino de Santiago, a partir de la información cuantitativa obtenida en una encuesta de satisfacción. Los resultados revelan una percepción global positiva y descartan problemas de percepción de saturación vinculados al creciente número de peregrinos que recorren sus localidades. Además, la valoración global no depende de la proximidad de la residencia al Camino, pero es más positiva para los individuos con ingresos vinculados a actividades turística y para los que tienen un contacto más frecuente con los peregrinos.

Palabras clave: Camino de Santiago; percepción residente; impacto socioeconómico.

Fecha de recepción: 11 de junio de 2019

Fecha de aceptación: 10 de enero de 2020

1 Este trabajo se benefició de la financiación procedente del Programa de Axudas para a Consolidación e Estruturación de Unidades de Investigación Competitivas del Sistema Universitario Gallego, Xunta de Galicia, en la modalidad de Grupos de Referencia Competitiva (ED431C 2017/44) y del Convenio de Colaboración entre la Agencia Turismo de Galicia y la Universidade de Santiago de Compostela.

** GAME-IDEGA y Departamento de Fundamentos da Análise Económica. Universidade de Santiago de Compostela. Avenida das Ciencias, Chalet ${ }^{\circ}$ 1. Campus Vida, USC. 15782 SANTIAGO DE COMPOSTELA (España).E-mail: melchor.fernandez@usc.es,di.fernandez.mendez@usc.es, dolores.riveiro@usc.es 


\title{
The local impact of the St James Way: the perception of residents from two Galician municipalities
}

\begin{abstract}
This study explores the perception of residents from two rural municipalities on impacts deriving from the St James Way. The analysis is conducted on the basis of the quantitative information obtained from a satisfaction survey. The results reveal a positive overall perception, ruling out saturation perception problems linked to the increasing number of pilgrims walking along their communities. Moreover, global perception does not depend on the proximity of the individual's residence to the Way but is more positive for individuals deriving income from tourism activities and for those having more intensive contact with pilgrims.
\end{abstract}

Keywords: St James Way; residents' perception; socioeconomic impact.

\section{INTRODUCCIÓN}

Con 1.200 años de historia, el Camino de Santiago no es sólo la imagen más conocida de Galicia, sino que es símbolo de la construcción y la identidad cultural europea. El flujo de peregrinos procedentes de toda Europa actuó como una verdadera "vía del conocimiento", que difundió por todo el recorrido los grandes movimientos culturales y artísticos surgidos en Europa. Al mismo tiempo, la peregrinación a Santiago de Compostela dejó una huella "física", materializada en una red de servicios dedicados a atender las necesidades de los peregrinos, (monumentos, iglesias, hospitales, albergues y hospederías), que ahora constituyen un patrimonio histórico y cultural de inestimable valor. Registrado como primer Itinerario Cultural Europeo en 1987 y como Patrimonio de la Humanidad por la UNESCO en 1993, la relevancia del Camino va más allá de la de un mero recurso turístico.

El Camino, cuyo origen es sin duda religioso, ha evolucionado, convirtiéndose en la actualidad en uno de los principales atractivos de Galicia y de España, y en un fenómeno económico que genera rentas y empleos, con la consiguiente dinamización y desarrollo de las localidades por donde discurre. El número de peregrinos que recogieron la Compostela (certificado que se expide como reconocimiento de la Iglesia por haber peregrinado hasta Santiago) en 2018 fue de 327.378, un nuevo récord absoluto. Quince años antes, en 2003, fueron 74.614, es decir, 252.764 menos, lo que supone una tasa de crecimiento anual acumulativa superior al $10 \%$. El éxito del Camino durante estos años es evidente y en cierto modo ha sido sorprendente. No se puede negar una nueva visión del Camino como atracción turística, con connotaciones culturales y espirituales, que está siendo protegido, promocionado y comercializado tanto por instituciones públicas como privadas. Ante la amenaza de que el Camino llegue a convertirse en una gran arteria turística de masas, defender su sostenibilidad, manteniendo su esencia y globalizando su disfrute, es un objetivo para todos los agentes implicados.

Para gestionar mejor los retos derivados de la creciente demanda en el Camino de Santiago, es esencial comprender la percepción y actitudes de los residentes con respecto a 
los peregrinos. Son múltiples las relaciones que se establecen entre peregrinos y residentes, y aunque estas se entablan en un contexto no directamente turístico, tienen importantes similitudes en muchos aspectos. Por ello, el impacto de la afluencia de peregrinos para las poblaciones locales puede ser analizado en términos de lo que la literatura establece, en general, para cualquier recurso turístico. La comunidad local puede ser el mejor defensor de la esencia y sostenibilidad del Camino o un elemento que puede provocar el declive del recurso. Por ello, conocer la actitud del residente es fundamental y, de hecho, se ha situado como una de las cuestiones más abordadas en el campo de la investigación en turismo, especialmente en las últimas tres décadas.

Tradicionalmente, los estudios sobre la actividad turística en los diferentes destinos se han centrado más en la satisfacción de los visitantes que en la de los residentes (Castillo et al., 2012). Es a partir de la década de 1970, cuando se empiezan a tener en cuenta los intereses y la percepción de la población residente en los análisis del turismo, una vez que la actividad turística es entendida como un acontecimiento sociocultural en el que se encuentran turistas y residentes y donde se generan efectos para las poblaciones locales (Murphy, 1985; Harril, 2004). De acuerdo con la literatura, la actividad turística es un elemento de dinamización económica para las poblaciones locales, pero también genera impactos - positivos y negativos - en otras dimensiones como la sociocultural o la medioambiental, que influyen en la calidad de vida de los residentes (Brida et al., 2014; Deery et al., 2012; Vargas-Sánchez et al., 2011; Andereck et al., 2005; Easterling, 2004; Gursoy et al., 2002; Tosun, 2002; Mathiesony Wall, 1982). En cualquier caso, se confirma que la actitud y el apoyo de la población residente a las actividades turísticas es un elemento determinante en el éxito y la sostenibilidad de estas actividades (Lee 2013; Nicholas et al., 2009; VargasSánchez et al., 2009; Aguiló y Roselló, 2005; Jurowski y Gursoy, 2004; Andriotis y Vaughan, 2003; Gursoy et al., 2002; Sheldon y Abenoja, 2001; Garrod y Fyall, 1998; Ap, 1992).

En el caso del Camino de Santiago, cabe esperar que la presencia de peregrinos, y su interacción con el territorio que recorren y con la población residente, genere impactos importantes, lo que ha sido objeto de análisis en numerosos trabajos que en su gran mayoría se han focalizado en el estudio del impacto del Camino sobre el sector turístico y su potencialidad como factor de desarrollo local (Precedo et al, 2007; Maak, 2009; Andrade et al, 2010; Andrade, 2011; Porcal et al., 2012; Martín Duque, 2014; Pardellas y Padín, 2015; Martín Duque, 2017; Lois-González et al., 2018). En cuanto a los análisis de percepción, la visión de los peregrinos es estudiada desde hace años a partir de la información de varias encuestas de satisfacción con la experiencia ${ }^{2}$, pero existen muy pocos estudios sobre la percepción de los residentes y que, en la mayoría de los casos, no son específicos sobre el Camino sino sobre la actividad turística en general (Gigirey, 2003; Santos-Solla y López, 2015). ${ }^{3}$

2 La Federación Española de Asociaciones de Amigos del Camino de Santiago publica cada año en la revista Peregrinos los resultados de una encuesta específica sobre la percepción que los peregrinos tienen del Camino. También aporta información sobre la percepción o la satisfacción de los peregrinos con el Camino, aunque no es específica, la "Encuesta de Turismo en Destino de Galicia" (ETDG) que realiza Turgalicia.

3 Este también es el caso de la "Encuesta de valoración y percepción del turismo en la ciudad de Santiago de Compostela", realizada por el Observatorio Turístico de Santiago de Compostela, que 
El objetivo de este trabajo es explorar la percepción de los residentes sobre los impactos, positivos y negativos, que se derivan de la existencia del Camino y de la afluencia de peregrinos a nivel global y en los ámbitos sociocultural, económico y ambiental, prestando especial atención a la percepción de saturación y a la actitud de los residentes hacia el Camino. Comprobar cuál es el apoyo a escala local de la evolución actual del Camino y caracterizar los aspectos más problemáticos desde el punto de vista de los residentes, son elementos esenciales para una correcta concienciación sobre la capacidad de la cultura, el arte y el patrimonio natural como factores esenciales para el desarrollo económico local desde la perspectiva de la sostenibilidad. En muchas localidades rurales por las que discurre el Camino, durante buena parte del año el flujo diario de visitantes es superior al volumen de residentes. ${ }^{4}$ Siendo así, el éxito del Camino, y el de las políticas para su desarrollo, no debe ser valorado sólo por la aceptación por parte de los peregrinos, que podemos medir por el crecimiento de los flujos anuales, sino por la aprobación por parte de la población que reside en los municipios que recorre.

La información cuantitativa se obtuvo a partir de una encuesta sobre la percepción de los residentes, realizada en Pedrafita do Cebreiro y Melide, dos municipios representativos del tramo gallego del Camino Francés, que se corresponde con un área rural en declive social y económico. Los resultados muestran que los residentes perciben un impacto positivo del Camino en las distintas dimensiones y tienen una actitud favorable hacia el Camino y los peregrinos, resultado que, con alguna excepción como el trabajo de Jonhson et al. (1994), también se alcanza en la mayoría de los trabajos realizados para la percepción de impactos del turismo en comunidades muy diversas (Andereck et al., 2005). La percepción de impactos positivos, por si sola, no siempre explica la aceptación de un mayor desarrollo turístico (Muler González et al., 2018); si bien es el principal determinante del apoyo al desarrollo turístico (Rasoolimanesh et al., 2015).

A partir de esta introducción, el trabajo se estructura como sigue. El apartado dos revisa la literatura sobre los impactos del turismo y los principales factores determinantes de la percepción de los residentes en las distintas dimensiones del impacto. En el apartado tres se describen las principales características de los municipios objeto de estudio y la afluencia de peregrinos en los últimos años. En el apartado cuatro, se explica la estructura de la encuesta y la metodología del análisis. En el apartado cinco se presentan y discuten los resultados y, finalmente, el apartado seis presenta las principales conclusiones.

estudia el conocimiento y valoración que los residentes hacen del sector turístico, no del impacto que le atribuyen sobre su bienestar.

http://www.santiagoturismo.com/files/2016/02/ENQUISAPOBOACINLOCAL2005.pdf

4 La carga de población vinculada al turismo en Santiago de Compostela supone el 5\% de los residentes. En municipios rurales del Camino Francés como Portomarín, Pedrafita do Cebreiro o Triacastela, el peso es muy superior, un $29 \%, 19,3 \%$ y 18,2\%, respectivamente. (IGE, Carga de población estacional de los municipios de Galicia https://www.ige.eu/web/mostrar_actividade_estatistica.jsp?idioma $=$ es\& codigo $=0201001008$ ). 


\section{FACTORES QUE INFLUYEN EN LA PERCEPCIÓN Y LA ACTITUD DE LOS RESIDENTES HACIA EL TURISMO: UN BREVE REPASO DE LA EVIDEN- CIA DISPONIBLE}

Entre las teorías más utilizadas para explicar cómo se originan las actitudes de los residentes hacia los impactos del turismo se encuentra la Teoría del Intercambio Social (Gursoy y Rutherford, 2004). Esta aproximación concluye que la actitud positiva o negativa de la población local hacia el turismo y su apoyo al desarrollo turístico depende de la percepción de los impactos positivos y negativos de la actividad (Homans 1961; Ap, 1992). De acuerdo con esta teoría, los residentes evalúan los beneficios y los costes derivados de la actividad turística y, cuanto más positivo sea el balance, más favorable será su actitud (Pizam, 1978; Ap, 1990; Allen et al., 1993; Jurowski et al., 1997; Gursoy et al., 2002; Sharpley, 2014).

\subsection{Percepciones de impacto y actitudes}

En la literatura se reconoce que los residentes perciben impactos en distintas dimensiones (económicos, sociales, culturales y medioambientales), pero que los beneficios económicos percibidos suelen ser los principales determinantes de la actitud positiva hacia el turismo (Rasoolimanesh et al., 2015; Abdollahzadeh y Sharifzadeh, 2014; Castillo et al., 2012; Díaz y Gutiérrez, 2010; Gursoy et al., 2009; Akis et al., 1996; Perdue et al., 1990). Los beneficios económicos derivados de las actividades turísticas están relacionados, principalmente, con nuevas oportunidades de empleo (Vargas et al., 2009; Haley et al., 2005; Tosun, 2002; Willians y Lawson, 2001; Davis et al., 1988; Tyrrell y Spaulding, 1984), mayores ingresos (Murphy, 1981; Davies et al., 1988; Lankford, 1994; Tyrrell y Spaulding, 1984) y nuevas oportunidades de negocio (McGehee y Andereck, 2004; Davis et al., 1988).Por su parte, los costes económicos se relacionan, en gran medida, con los incrementos de los precios de bienes y servicios, incluida la vivienda, (Fraumany Banks, 2011; Haley et al., 2005; Jurowski y Gursoy, 2004).

En la dimensión sociocultural, los beneficios se asocian, sobre todo, al aumento de las actividades recreativas y de ocio para los residentes (Kim et al., 2013; Tovar y Lockwood, 2008; Haley et al., 2005; Gursoy et al., 2002; Allen et al., 1993; Sheldom y Var, 1984; Murphy, 1981; Pizam, 1978), la mejora de los servicios e infraestructuras (Kim et al., 2013; Tovar and Lockwood, 2008; Andereck et al., 2005; Andreotis, 2005; Shariff y Tahir, 2003; Willians y Lawson, 2001; Pizam, 1978), las oportunidades de intercambio cultural que ofrece (Kim et al., 2013; Andereck et al., 2007; Willians y Lawson, 2001) y la preservación de la identidad cultural y la revitalización de las tradiciones (Besculides et al., 2002; Kreag, 2001; Haralambopoulos y Pizam, 1996). Todo ello redunda en una mejora en la calidad de vida de los residentes, que es en lo que se traducen, en última instancia, los beneficios sociales del turismo (Brunt y Courtney, 1999). Pero el turismo también genera costes sociales (Andereck et al., 2005; Tosun, 2002; Brunt y Courtney, 1999). Las actitudes negativas hacia el turismo parecen estar asociadas a ciertos aspectos relacionados con la insatisfacción con el acceso a servicios públicos (Brunt y Courtney, 1999; Allen et al., 1988; Chen, 2011), los problemas de masificación y congestión (Andereck et al., 2005; Liu y Var, 1986; Tyrrell, 1984) y el 
incremento de las actividades delictivas (Andereck et al., 2007, 2005; Wall y Mathieson, 2006; Haley et al., 2005). Además, el turismo puede tener otros efectos negativos como el deterioro del patrimonio cultural (Gursoy et al., 2002; Chen, 2011) o la pérdida del carácter y la cultura tradicional de localidades pequeñas (Choi y Murray, 2010; Johnson et al., 1994), además de crear conflictos sociales, tanto entre residentes como entre residentes y turistas (Choi y Murray, 2010; Tosun, 2002).

La preocupación por cuestiones medioambientales también influye en la percepción que los residentes tienen del impacto del turismo (Gursoy et al., 2002; Hillery et al., 2001; Jurowski et al., 1997). De acuerdo con Jurowski et al. (1997), los residentes tienden a evaluar los impactos económicos y sociales de forma positiva y los medioambientales de forma negativa. La actividad turística puede incrementar y encarecer la gestión de residuos (Andereck et al., 2005; Haley et al., 2005; Jurowski y Gursoy, 2004; Brunt y Courtney, 1999) y puede afectar negativamente al medio natural (Choi y Murray, 2010; Mathiesony Wall, 1982), además de alterar la apariencia física del área (Willians y Lawson, 2001). Sin embargo, los residentes también pueden percibir que el turismo no contribuye al deterioro medioambiental (Liu y Var, 1986) e, incluso, que es una razón importante para proteger los recursos naturales (Díaz y Gutiérrez, 2010).

\subsection{Principales factores de estudio en el análisis de las percepciones de los residentes}

La percepción que tienen los residentes de los beneficios y los costes del turismo en un destino puede verse influida por diversos factores, relacionados con su perfil sociodemográfico y económico, así como con su relación con el sector turístico y los turistas (Easterling, 2004 y Deery et al. 2012 ofrecen una revisión). Junto a las características sociodemográficas, como el nivel educativo, el nivel de renta, la edad o el género (Abdollahzadeh y Sharifzadeh, 2014; Sharma y Dyer, 2009; Haley et al., 2005; Andereck et al., 2005; McGehee y Andereck, 2004; Tosun, 2002; Williams y Lawson, 2001; Johnson et al., 1994; Lankford, 1994; Liu y Var, 1986), destacan algunos factores como la dependencia económica del turismo. Si bien la percepción no suele ser muy diferente según las características sociodemográficas, la evidencia muestra que los individuos cuyos ingresos están vinculados a la actividad turística tienen una percepción de los impactos del turismo más positiva (Andereck et al., 2007; Andereck et al., 2005; Andriotis, 2005; Haley et al., 2005; Akis et al., 1996; Jonhson et al., 1994; Perdue et al., 1990). Otros factores, como la intensidad del contacto con los turistas (Andereck et al., 2007; Akis et al.,1996; Lankford y Howard, 1994) y la proximidad a la zona turística (Haley et al., 2005; Jurowski y Gursoy, 2004; Harril, 2004; Sheldon y Var, 1984), también influyen en la percepción de los residentes. A este respecto, algunos trabajos concluyen que cuanto mayor es la proximidad del residente al recurso turístico más positiva es la percepción de impacto (Keogh, 1990; Belisle y Hoy, 1980); mientras que otros encuentran que son los que viven próximos al recurso turístico los que perciben menores impactos positivos y tienen una actitud menos favorable hacia el turismo (Willians y Lawson, 2001; Madrigal, 1993; Pizam, 1978). De acuerdo con Jurowski y Gursoy (2004), el mayor uso de los recursos turísticos por parte de los residentes que viven próximos a esos recursos podría explicar esos resultados. En ese sentido, Chen, 2011 concluye que la percepción es más positiva cuando la actividad turística consiste en eventos de los que también participan los residentes. 
Por otra parte, también pueden influir en la percepción de los impactos factores relacionados con el "apego" o la "identificación" con la comunidad, medida por el tiempo de residencia en la localidad o haber nacido allí (Almeida-García et al., 2016; Jurowski et al., 1997; Sheldon y Var, 1984) o la preocupación e interés por la comunidad (Gursoy et al., 2002). Mientras algunos trabajos encuentran que cuanto mayor es el "apego" más negativa es la percepción (Lankford y Howard, 1994), en otros no se concluye que ese sea el caso general (Andereck et al., 2005; Gursoy et al., 2002). Además, aspectos como la dependencia que la localidad tiene del turismo (Haralambopoulos y Pizam, 1996; Johnson et al, 1994; Long et al., 1990; Liu y Var, 1986) o el nivel de desarrollo local, influyen también en la percepción de impacto. En lo relativo a este segundo aspecto, varios estudios concluyen que es probable que los residentes de zonas más deprimidas infravaloren los costes y sobreestimen los beneficios económicos del turismo (Liu y Var, 1986; Sheldon y Var, 1984).

\section{PEREGRINOS Y RESIDENTES EN EL CAMINO DE SANTIAGO}

\subsection{Algunos rasgos de la evolución reciente del Camino de Santiago}

El éxito del Camino a escala global es una realidad que se refleja en el incremento continuo del número de peregrinos que deciden llegar hasta Santiago de Compostela. Tradicionalmente los récords de peregrinos se producían en los años santos, el último en 2010, pero la popularidad del Camino de Santiago fuera de España, donde cada vez más es un destino de aventura, cultural y espiritual muy valorado, ha supuesto que no haya sido necesario llegar al próximo año santo (2021) para lograr superar las cifras de peregrinos de 2010. Pero no solo ha aumentado la cifra de peregrinos, también ha variado la afluencia en las diferentes estaciones del año (menores diferencias entre la temporada alta y la temporada baja), las rutas elegidas o la relevancia de los peregrinos extranjeros.

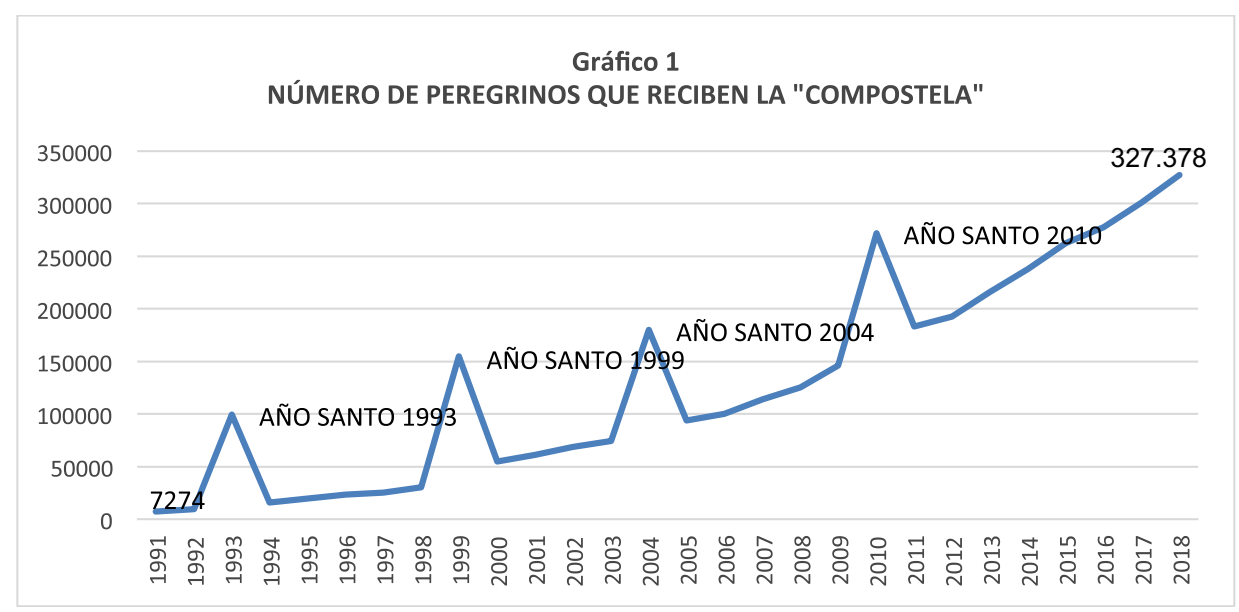

Fuente: Elaboración propia en base a los datos de los Informes estadísticos de la Oficina del Peregrino. 
Para comprender como ha evolucionado el Camino de Santiago mostremos algunas cifras. En 2018 recogieron la Compostela ${ }^{5}$ un $82 \%$ más de peregrinos que en 2004 y un $20 \%$ más que en 2010. El aumento de los peregrinos extranjeros es especialmente significativo, más de un $320 \%$ desde 2004 y con más de 146 nacionalidades representadas. Es también muy llamativo el crecimiento continuo de algunas rutas consideradas secundarias. El Camino Francés sigue siendo la alternativa elegida por la mayoría de los peregrinos (57\%), aunque su dominio es cada vez menor por el fuerte crecimiento del Camino Portugués o el Camino Inglés. En sólo 14 años, el Camino Francés ha perdido algo más de 20 puntos porcentuales de cuota. No obstante, el incremento global es tan fuerte que, a pesar de esta pérdida de cuota, el Camino Francés ha aumentado año a año sus cifras de peregrinos $(2,12 \%$ anual desde 2004) aunque sin superar aun el récord de peregrinos alcanzado en el último año santo (2010).

\subsection{Descripción del área geográfica}

El Camino es una realidad que destaca por su diversidad. Galicia, sumando las diferentes rutas alternativas, cuenta con aproximadamente $1.500 \mathrm{Km}$ de recorrido del Camino de Santiago, que atraviesa mayoritariamente áreas rurales de muy baja densidad de población, pero con características, geográficas, físicas, medioambientales y sociales, muy diversas. Este hecho y que el peregrino actual no obedece a un perfil homogéneo (diferencias en edad, nacionalidad, nivel educativo, renta, motivación...) que puede diferir en función de la ruta realizada, impide realizar un análisis del impacto del Camino que sea generalizable a todas las localidades que atraviesa. Por ello, se ha optado por realizar el estudio en dos áreas rurales representativas del tramo gallego del Camino Francés, donde teóricamente la intensidad del impacto debería ser más relevante, tanto económica como socialmente.

En concreto, el estudio se ha realizado en Pedrafita do Cebreiro (tramo anterior a Sarria) y Melide (en el tramo posterior a Sarria, Figura 1). Teniendo como elemento común la continua pérdida de población (aunque mucho más intensa en Pedrafita do Cebreiro), los municipios seleccionados tienen diferencias geográficas, socioeconómicas y demográficas importantes que los situarían en posiciones de partida casi opuestas. Pedrafita es un municipio de alta montaña de la provincia de Lugo, en la frontera con Castilla-León, con poco más de 1.000 habitantes, con la estructura demográfica más envejecida del Camino Francés (la edad media es de 57,38 años), la menor densidad de población (10,4 habitantes por $\mathrm{Km}^{2}$ ) y un poblamiento muy disperso (en su núcleo principal vive solo el $18 \%$ del total de la población del municipio). Por su parte, Melide se encuentra en el centro de Galicia, en la provincia de A Coruña, y cuenta con casi 7.500 habitantes. Dentro del trazado del Camino Francés, es el municipio más “joven” (con una edad media de 47,56 años) y el más urbano (aunque cuenta con 189 núcleos de población, lo que supone de partida una elevada dispersión de la población, en su núcleo principal viven más de 4.500 personas, el $60 \%$ de la población total del municipio). Pero seguramente su principal diferencia en relación al análisis planteado es que el número de peregrinos que recorre cada tramo

5 La Compostela es un certificado expedido por la iglesia como reconocimiento de haber peregrinado hasta Santiago por motivos religiosos o espirituales. Requiere hacer a pie o a caballo los últimos $100 \mathrm{Km}$ o los últimos 200 en bicicleta. 
es muy diferente cuantitativa y cualitativamente. Con datos de 2017, la cifra estimada de peregrinos que pasaron por el municipio de Pedrafita do Cebreiro es de algo más de 90.000 , mientras que para Melide esa cifra superó ampliamente el doble. ${ }^{6}$ Estos números suponen en términos absolutos intensidades muy diferentes en ambos territorios (mientras que para Pedrafita do Cebreiro los peregrinos suponen una población equivalente anual de 250 personas en Melide son más del doble, 515). Pero al poner estas cifras en relación con la población censada en cada municipio, vemos que el impacto relativo sobre cada localidad es también muy diferente: mientras en Pedrafita los peregrinos suponen un 23\% del total de población, en Melide son sólo un 6,9\%.

\section{Figura 1 \\ MUNICIPIOS DEL CAMINO FRANCÉS EN EL TRAMO GALLEGO}

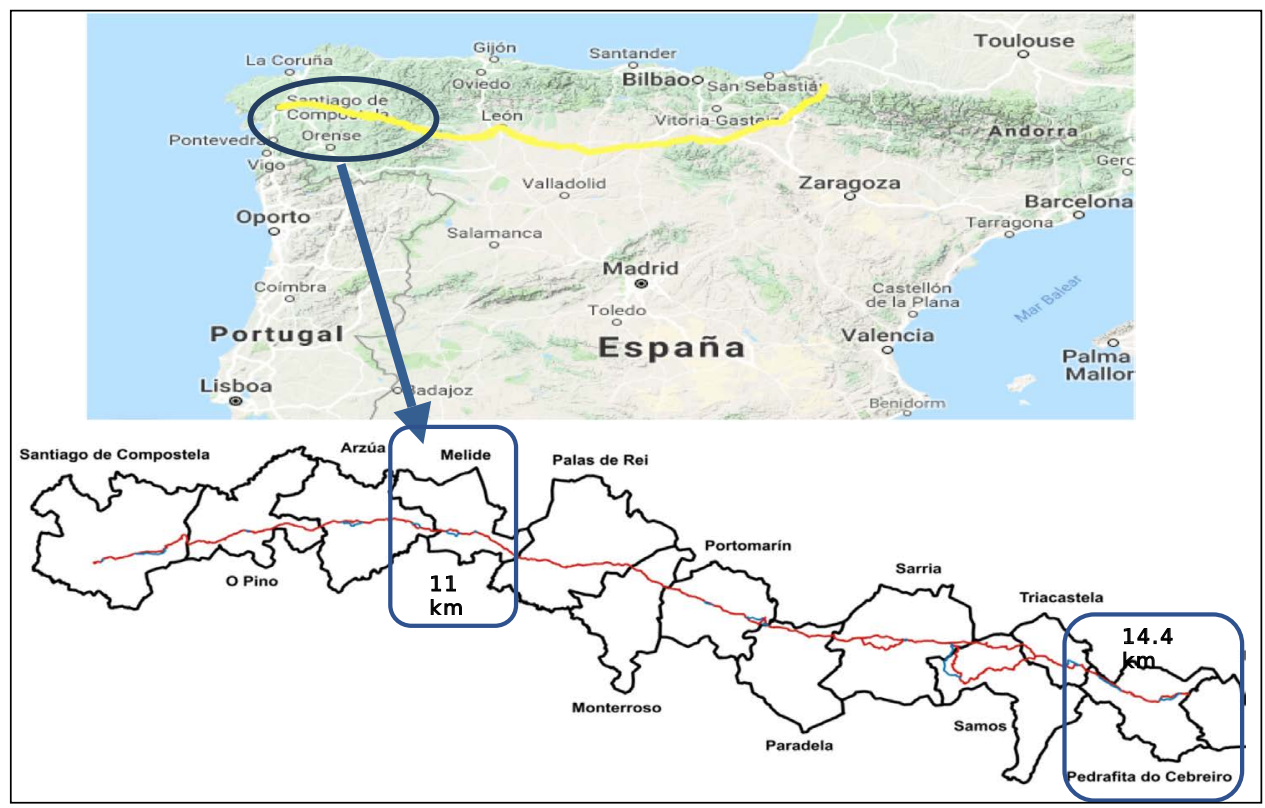

Fuente: Elaboración propia.

En resumen, Pedrafita do Cebreiro es un municipio rural poco poblado, que lleva años perdiendo población (solo dos nacimientos en 2017 y una tasa anual acumulativa de crecimiento desde el año 2000 de $-2,45 \%$ ) y con una fuerte especialización en el sector primario (26\% de los afiliados). Melide es una cabecera comarcal considerada

6 Aunque en Melide se unen los Caminos Primitivo y Francés, lo que supone un aumento considerable de peregrinos respecto a las localidades anteriores, el enorme diferencial respecto a Pedrafita do Cebreiro se explica porque un alto porcentaje de peregrinos (mayoritariamente nacionales) inician el Camino en Sarria para asegurar realizar el mínimo de Km exigible para "merecer" la Compostela. En concreto, en 2017, el 44\% de los peregrinos que realizaron el Camino Francés comenzaron su andadura en Sarria. 
como zona intermedia según el grado de urbanización y con una fuerte especialización en el sector servicios (68\% de los afiliados). En ambos casos, la Hostelería emplea a un porcentaje creciente de población (un 17\% del total de afiliados en 2017). Si no tenemos en cuenta a los trabajadores autónomos, entre los que el peso de las actividades primarias sigue siendo muy elevado, la Hostelería es la principal fuente de empleo en ambos municipios. Diez años antes, en 2007, la Hostelería ocupaba a menos del 9\% de los trabajadores, siendo la cuarta actividad en términos de capacidad de generación de empleo.

\section{DATOS Y METODOLOGÍA}

\subsection{Muestra}

Los datos primarios para el análisis fueron obtenidos a partir de una encuesta de satisfacción realizada a los residentes de Pedrafita do Cebreiro y Melide de 18 y más años. La muestra diseñada es estratificada proporcional, siendo los estratos considerados los grandes grupos de edad y la localización de la parroquia de residencia con relación al Camino Francés. Al ser poblaciones envejecidas, y dada la menor propensión de las personas mayores a responder encuestas, consideramos relevante tener en cuenta la edad en el diseño muestral para evitar este sesgo. Se tomaron tres tramos de edad (1839; 40-64; 65), a partir del total de población de entre 18 y 75 años de acuerdo con el Padrón Municipal de Habitantes de 2016 (782 en Pedrafita do Cebreiro y 5.396 en Melide). En cuanto a la estratificación por parroquias, este es el ámbito territorial en la que se dividen los municipios en Galicia, y no por todas las parroquias de estos municipios pasa el Camino. Cabe esperar que los residentes en parroquias del Camino tendrán un mayor contacto con los peregrinos y una mayor percepción de los impactos. Además, en la encuesta piloto realizada se detectó que los residentes en parroquias de fuera del Camino, si bien perciben impactos para el municipio, se sienten menos concernidos y muestran menor propensión a responder encuestas sobre este asunto. La muestra diseñada para Pedrafita do Cebreiro asciende a 258 y para Melide a 359. Una vez establecida la distribución de la muestra según los estratos considerados (edad y parroquia de residencia), las encuestadoras seleccionaron a las personas a encuestar hasta alcanzar la cuota correspondiente en cada caso. En el caso de Pedrafita do Cebreiro, de la muestra inicialmente calculada, finalmente se realizaron 212 , es decir, un $82,17 \%$ del objetivo inicial; lo que implica que el error máximo de muestreo es del 5,75\%. Para Melide, el número de encuestas finalmente realizadas fue de 260 , un $72,43 \%$ de la muestra diseñada, siendo el error máximo de muestreo del 5,93\%. Las encuestas fueron realizadas personalmente, por encuestadoras con formación, durante el mes de agosto y la primera semana de septiembre de 2017. En la Tabla 1 se resumen los principales estadísticos descriptivos de la muestra realizada. Los resultados son consistentes con la información disponible en el Censo de Población.

La distancia, además de aproximarla por la situación de la parroquia de residencia, podemos valorarla a partir de lo que reportan los encuestados. En el caso de Pedrafita, algo más de la mitad viven a menos de diez minutos del Camino (dado que no se especificaba, posiblemente en muchos casos entendieron que era el tiempo de desplazamiento 
en coche), mientras en Melide están en ese caso la inmensa mayoría (85,4\%), lo cual es razonable al pasar el Camino por la capital del municipio. En cuanto a la intensidad del contacto con los peregrinos, mientras en Pedrafita, un 20,3\% dice no tener ningún contacto, en Melide esto sólo ocurre en el 2\% de los casos. En el otro extremo, los que tienen contacto varias veces al día, son un $25 \%$ en Pedrafita y casi el $69 \%$ en Melide, lo cual es consistente con que un $60,8 \%$ de los encuestados señalen que la razón por la que tienen contacto con los peregrinos es porque pasan por su lugar de residencia.

Tabla 1

ESTADÍSTICOS DESCRIPTIVOS DE LA MUESTRA REALIZADA

\begin{tabular}{|c|c|c|c|c|c|}
\hline \multirow[t]{2}{*}{ Variable } & \multirow[t]{2}{*}{ Categorías } & \multicolumn{2}{|c|}{ Pedrafita do Cebreiro } & \multicolumn{2}{|c|}{ Melide } \\
\hline & & Frecuencia & Porcentaje & Frecuencia & Porcentaje \\
\hline \multirow{2}{*}{$\begin{array}{l}\text { Parroquia } \\
\text { del Camino }\end{array}$} & $\mathrm{Si}$ & 109 & $51 \%$ & 222 & $85 \%$ \\
\hline & No & 103 & $49 \%$ & 38 & $15 \%$ \\
\hline \multirow[t]{4}{*}{ Edad } & $18-39$ & 70 & $33 \%$ & 96 & $37 \%$ \\
\hline & $40-64$ & 106 & $50 \%$ & 132 & $51 \%$ \\
\hline & 65 & 36 & $17 \%$ & 31 & $12 \%$ \\
\hline & NS / NC & 0 & $0 \%$ & 1 & $0,4 \%$ \\
\hline \multirow[t]{2}{*}{ Género } & Hombre & 106 & $50 \%$ & 118 & $45 \%$ \\
\hline & Mujer & 106 & $50 \%$ & 142 & $55 \%$ \\
\hline \multirow{4}{*}{$\begin{array}{l}\text { Nivel de } \\
\text { estudios }\end{array}$} & Primarios & 100 & $47 \%$ & 86 & $33 \%$ \\
\hline & Secundarios & 63 & $30 \%$ & 87 & $33,5 \%$ \\
\hline & Superiores & 49 & $23 \%$ & 86 & $33 \%$ \\
\hline & $\mathrm{NS} / \mathrm{NC}$ & 0 & $0 \%$ & 1 & $0,4 \%$ \\
\hline \multirow{4}{*}{$\begin{array}{l}\text { Tiempo de } \\
\text { residencia } \\
\text { al año en } \\
\text { municipio }\end{array}$} & Habitualmente & 145 & $68 \%$ & 223 & $86 \%$ \\
\hline & Vacaciones ( $<3$ meses) & 25 & $12 \%$ & 27 & $10 \%$ \\
\hline & Fines de semana & 34 & $16 \%$ & 9 & $3 \%$ \\
\hline & Ocasionalmente & 8 & $4 \%$ & 1 & $0 \%$ \\
\hline \multirow{5}{*}{$\begin{array}{l}\text { Situación } \\
\text { laboral }\end{array}$} & \begin{tabular}{|l|} 
Jubilado \\
\end{tabular} & 47 & $22 \%$ & 35 & $13 \%$ \\
\hline & Estudiante & 3 & $1 \%$ & 9 & $4 \%$ \\
\hline & Desempleado & 13 & $6 \%$ & 19 & $7 \%$ \\
\hline & Ocupado & 144 & $68 \%$ & 187 & $72 \%$ \\
\hline & Otras & 5 & $3 \%$ & 10 & $4 \%$ \\
\hline \multirow{5}{*}{$\begin{array}{l}\text { Sector de } \\
\text { actividad de } \\
\text { ocupados }\end{array}$} & Primario & 24 & $17 \%$ & 17 & $9,1 \%$ \\
\hline & Secundario & 9 & $6 \%$ & 11 & $5,9 \%$ \\
\hline & Construcción & 4 & $3 \%$ & 4 & $2,1 \%$ \\
\hline & Servicios & 107 & $74 \%$ & 154 & $82,4 \%$ \\
\hline & NS / NC & 0 & $0 \%$ & 1 & $0,5 \%$ \\
\hline \multirow{3}{*}{$\begin{array}{l}\text { Distancia } \\
\text { residencia al } \\
\text { Camino }\end{array}$} & $<10$ minutos & 116 & $55 \%$ & 214 & $82 \%$ \\
\hline & Entre 10 y 30 minutos & 62 & $29 \%$ & 30 & $12 \%$ \\
\hline & $>30$ minutos & 34 & $12 \%$ & 16 & $6 \%$ \\
\hline
\end{tabular}




\begin{tabular}{|l|l|r|r|r|r|}
\hline \multirow{2}{*}{ Variable } & Categorías & \multicolumn{2}{|c|}{ Pedrafita do Cebreiro } & \multicolumn{2}{|c|}{ Melide } \\
\cline { 2 - 6 } & & Frecuencia & Porcentaje & Frecuencia & Porcentaje \\
\hline \multirow{2}{*}{$\begin{array}{l}\text { Frecuencia } \\
\text { contacto con } \\
\text { peregrinos }\end{array}$} & Ninguno & 43 & $20 \%$ & 4 & $2 \%$ \\
\cline { 2 - 6 } & Pocas veces & 90 & $42 \%$ & 47 & $18 \%$ \\
\cline { 2 - 6 } & Alguna vez cada día & 27 & $13 \%$ & 30 & $12 \%$ \\
\cline { 2 - 6 } & Varias veces cada día & 52 & $25 \%$ & 179 & $69 \%$ \\
\hline \multirow{3}{*}{$\begin{array}{l}\text { Motivo del } \\
\text { contacto }\end{array}$} & Trabajo & 51 & $30,2 \%$ & 72 & $28 \%$ \\
\cline { 2 - 6 } & $\begin{array}{l}\text { Pasan por localidad } \\
\text { residencia }\end{array}$ & 62 & $36,7 \%$ & 158 & $61 \%$ \\
\cline { 2 - 6 } & En otra localidad & 55 & $32,5 \%$ & 30 & $12 \%$ \\
\cline { 2 - 6 } & NS / NC & 1 & $0,6 \%$ & 0 & $0 \%$ \\
\hline
\end{tabular}

Fuente: Elaboración propia.

En resumen, los estadísticos descriptivos ofrecen importantes diferencias entre municipios, algunas ya conocidas por la información disponible en el Censo, (como la mayor dotación de capital humano en Melide o la relevancia del sector primario en Pedrafita do Cebreiro), y otras que podían intuirse dado el trazado del camino en ambos municipios, que con recorridos de longitudes similares, tienen importantes diferencias en relación a la distancia de la residencia habitual al Camino y, sobre todo, en relación al contacto con los peregrinos, muy superior en Melide.

\subsection{Cuestionario}

El cuestionario diseñado para medir la percepción de los impactos del Camino, que está dividido en tres partes, incluye los ítems que, en general, se derivan de la literatura revisada en el apartado 2. La selección final de las preguntas tuvo en cuenta la validación por un grupo de expertos y los resultados preliminares de una encuesta piloto. La primera parte es la más extensa e incluye 34 afirmaciones relacionadas con la percepción de los impactos (positivos y negativos) en las dimensiones económica, sociocultural, ambiental y global; así como con la actitud de los residentes respecto del camino. ${ }^{7}$ Se les pidió a los entrevistados que señalaran su nivel de acuerdo con cada afirmación usando una escala tipo Likert de 5 puntos (de 1, totalmente en desacuerdo, a 5 , totalmente de acuerdo). En relación con los impactos económicos percibidos, se analizan tanto los beneficios derivados del Camino para la actividad económica y el empleo como los costes (en términos de los efectos para el incremento del coste de la vida y el conflicto con el desarrollo de actividades no turísticas). Entre los aspectos por los que se pregunta en la dimensión social, se incluyen el impacto demográfico percibido, aspecto muy relevante en estos municipios con una dinámica poblacional regresiva, y la actitud general ante el Camino, indagando sobre el agrado que sienten los residentes

7 Una explicación más extensa del cuestionario y de los resultados descriptivos puede solicitarse a los autores. 
ante la presencia de peregrinos. En la dimensión medioambiental se busca conocer la percepción de los residentes sobre los impactos para el entorno natural y el paisaje, los núcleos de población, la dotación de infraestructuras básicas, la generación de residuos y la contaminación. En este bloque también se pregunta a los residentes por su percepción sobre la afluencia de peregrinos y, en concreto, si la consideran excesiva. En el último bloque de esta parte se obtiene una valoración global del encuestado sobre la percepción del impacto agregado del Camino, tanto desde el punto de vista de los efectos a nivel individual como a nivel del municipio.

La segunda parte trata de obtener información sobre la percepción que los residentes tienen, en general, del municipio en el que residen, pidiéndoles que identifiquen los tres principales problemas de una lista de 14, agrupados en cinco ámbitos: demográficos (despoblamiento, envejecimiento); económicos (falta de empleo y de oportunidades, precio de bienes y servicios); acceso a servicios (sociales, de salud, educativos, financieros, telecomunicaciones, etc.); culturales (falta de actividades culturales y de ocio) y otros problemas (afluencia de turistas/peregrinos, problemas de seguridad, conflictos entre vecinos y gestión del monte comunal). Por último, la tercera parte recoge información sobre las características sociodemográficas (edad, género, nivel de estudios y composición del hogar) y otros factores que, de acuerdo con la literatura, pueden influir en la percepción de los residentes; en particular, la intensidad del contacto con los peregrinos, la dependencia económica del turismo y la distancia del lugar de residencia al Camino (sobre la que se pregunta directamente y también se identifica a partir de la parroquia de residencia).

\section{RESULTADOS}

\subsection{Análisis descriptivo de variables intrínsecas}

La percepción de los encuestados para las distintas dimensiones del impacto es, en general y para los dos municipios considerados, positiva con diferencias poco significativas (Tabla 2).

Empezando el análisis por la dimensión económica (cuestiones 1-11), muy mayoritariamente, los residentes consideran que la existencia del Camino favorece los ingresos (más los del total de residentes que los propios), el comercio local, el empleo y el turismo; además de contribuir a que se mantengan otras actividades no turísticas. Por el contrario, los encuestados no perciben impactos económicos negativos, ni en términos de un perjuicio para el desarrollo de otras actividades económicas ni, en general, en términos de un incremento de los precios, salvo para el sector de hostelería en Pedrafita.

Respecto a otros impactos económicos, la percepción es diferente en los dos municipios analizados. En Pedrafita do Cebreiro, la percepción mayoritaria $(52,17 \%)$ es que el Camino es la principal actividad económica del municipio, pero que dicha actividad beneficia solo a un pequeño número de residentes. Por el contrario, los residentes de Melide no perciben mayoritariamente ni que el Camino sea la principal actividad económica del municipio ni que los beneficios de esa actividad reviertan en un pequeño número de residentes. Estos resultados son coherentes con la realidad de cada munici- 
pio, tanto en lo que respecta a sus características económicas como a la relevancia del número de peregrinos que reciben. En ambos casos, la gran mayoría no consideran que los beneficiarios sean residentes de fuera del municipio.

\section{Tabla 2}

\section{DESCRIPCIÓN DE LAS VARIABLES RELACIONADAS CON LA PERCEPCIÓN DE IMPACTO. FRECUENCIA Y (PORCENTAJE)}

\begin{tabular}{|c|c|c|c|c|c|c|c|}
\hline \multirow[b]{2}{*}{ Dimensión } & \multirow[b]{2}{*}{ İtems valorados " } & \multicolumn{3}{|c|}{ PEDRAFITA DO CEBREIRO } & \multicolumn{3}{|c|}{ MELIDE } \\
\hline & & $\mathbf{N}^{\mathrm{b}}$ & $\begin{array}{c}\text { Desacuerdo } \\
\text { (total o muy) }\end{array}$ & $\begin{array}{c}\text { Acuerdo } \\
\text { (total o muy) }\end{array}$ & $\mathbf{N}^{\mathrm{b}}$ & $\begin{array}{c}\text { Desacuerdo } \\
\text { (total o muy) }\end{array}$ & $\begin{array}{c}\text { Acuerdo } \\
\text { (total o muy) }\end{array}$ \\
\hline \multirow{15}{*}{$\begin{array}{l}\text { Impactos } \\
\text { Económicos }\end{array}$} & $\begin{array}{l}\text { 1. Mejora ingresos y nivel de vida de } \\
\text { residentes }\end{array}$ & 212 & $8(3,77 \%)$ & $199(93,87 \%)$ & 260 & $6(2,31 \%)$ & $248(95,38 \%)$ \\
\hline & $\begin{array}{l}\text { 2. Mejora ingresos y nivel de vida de la } \\
\text { familia }\end{array}$ & 212 & $89(41,98 \%)$ & $86(40,57 \%)$ & 260 & $111(42,69 \%)$ & $84(32,31 \%)$ \\
\hline & 3. Beneficia al comercio local & 212 & $4(1,89 \%)$ & $205(96,70 \%)$ & 260 & $9(3,46 \%)$ & $237(91,15 \%)$ \\
\hline & 4. Favorece al empleo & 212 & $24(11,32 \%)$ & $173(81,60 \%)$ & 258 & $14(5,43 \%)$ & $212(82,17 \%)$ \\
\hline & 5. Favorece al turismo & 212 & $2(0,94 \%)$ & $206(97,17 \%)$ & 260 & $10(3,85 \%)$ & $226(86,92 \%)$ \\
\hline & $\begin{array}{l}\text { 6. Favorece a otras actividades } \\
\text { económicas }\end{array}$ & 211 & $34(16,11 \%)$ & $140(66,35 \%)$ & 259 & $49(18,92 \%)$ & $157(60,62 \%)$ \\
\hline & 7. Entorpece las actividades no turísticas & 210 & $156(74,29 \%)$ & $35(16,67 \%)$ & 257 & $186(72,37 \%)$ & $39(15,18 \%)$ \\
\hline & 8. Aumentan los precios en & & & & & & \\
\hline & comercios & 212 & $83(39,15 \%)$ & $97(45,75 \%)$ & 258 & $148(57,36 \%)$ & $63(24,42 \%)$ \\
\hline & servicios & 212 & $105(49,53 \%)$ & $60(28,30 \%)$ & 259 & $146(56,37 \%)$ & $59(22,78 \%)$ \\
\hline & hosteleria & 212 & $66(31,13 \%)$ & $126(59,43 \%)$ & 260 & $121(46,54 \%)$ & $100(38,46 \%)$ \\
\hline & vivienda & 210 & $122(58,10 \%)$ & $49(23,33)$ & 256 & $194(75,78 \%)$ & $23(8,98 \%)$ \\
\hline & $\begin{array}{l}\text { 9. Principal actividad económica del } \\
\text { municipio }\end{array}$ & 207 & $83(40,10 \%)$ & $108(52,17 \%)$ & 252 & $147(58,33 \%)$ & $67(26,59 \%)$ \\
\hline & $\begin{array}{l}\text { 10. Favorece a residentes de fuera del } \\
\text { municipio }\end{array}$ & 211 & $174(82,46 \%)$ & $24(11,37 \%)$ & 249 & $158(63,45 \%)$ & $27(10,84 \%)$ \\
\hline & 11. Beneficia sólo a algunos residentes & 212 & $77(36,32 \%)$ & $122(57,55 \%)$ & 260 & $133(51,15 \%)$ & $101(38,85 \%)$ \\
\hline \multirow{10}{*}{$\begin{array}{l}\text { Impactos } \\
\text { Socio- } \\
\text { culturales }\end{array}$} & $\begin{array}{l}\text { 12. Favorece la creación o mejora de } \\
\text { servicios sanitarios }\end{array}$ & 212 & $120(56,60 \%)$ & $56(26,42 \%)$ & 255 & $146(57,25 \%)$ & $40(15,69 \%)$ \\
\hline & 13. Mejoran infraestructuras de transporte & 212 & $120(56,60 \%)$ & $61(28,77 \%)$ & 260 & $171(65,77 \%)$ & $39(15,00 \%)$ \\
\hline & $\begin{array}{l}\text { 14. Hay más actividades culturales y de } \\
\text { ocio }\end{array}$ & 211 & $132(62,56 \%)$ & $46(21,80 \%)$ & 253 & $86(33,99 \%)$ & $109(43,08 \%)$ \\
\hline & 15. Mejora el patrimonio cultural & 212 & $33(15,57 \%)$ & $161(75,94 \%)$ & 256 & $44(17,19 \%)$ & $166(64,84 \%)$ \\
\hline & $\begin{array}{l}\text { 16. Mejora calidad servicio en tiendas, } \\
\text { restaurantes }\end{array}$ & 212 & $92(43,40 \%)$ & $65(30,66 \%)$ & 259 & $86(33,20 \%)$ & $85(33,82 \%)$ \\
\hline & $\begin{array}{l}\text { 17. Oportunidad para conocer a otras } \\
\text { personas y culturas }\end{array}$ & 212 & $12(5,66 \%)$ & $180(84,91 \%)$ & 260 & $10(3,85 \%)$ & $223(85,77 \%)$ \\
\hline & $\begin{array}{l}\text { 18. Dificulta la movilidad y el acceso a } \\
\text { servicios públicos y privados }\end{array}$ & 212 & $169(79,72 \%)$ & $32(15,09 \%)$ & 257 & $167(64,98 \%)$ & $53(20,62 \%)$ \\
\hline & 19. Genera conflictos entre los residentes & 210 & $175(83,33 \%)$ & $19(9,05 \%)$ & 259 & $206(79,54 \%)$ & $19(7,34 \%)$ \\
\hline & $\begin{array}{l}\text { 20. Mayores efectos positivos si } \\
\text { peregrinos viajan solos/pareja que grupos }\end{array}$ & 212 & $42(19,81 \%)$ & $88(41,51 \%)$ & 258 & $75(29,07 \%)$ & $69(26,74 \%)$ \\
\hline & $\begin{array}{l}\text { 21. Mayores efectos negativos si } \\
\text { peregrinos viajan solos/pareja que grupos }\end{array}$ & 202 & $81(40,10 \%)$ & $9(4,46 \%)$ & 247 & $94(38,06 \%)$ & $17(26,74 \%)$ \\
\hline \multirow{5}{*}{$\begin{array}{l}\text { Impactos } \\
\text { medio- } \\
\text { ambientales }\end{array}$} & 22. Contribuye a preservar el paisaje & 212 & $50(23,58 \%)$ & $136(64,15 \%)$ & 259 & $42(16,22 \%)$ & $167(64,48 \%)$ \\
\hline & $\begin{array}{l}\text { 23. Contribuye a que los núcleos de } \\
\text { población estén más cuidados, limpios... }\end{array}$ & 212 & $72(33,96)$ & $117(55,19 \%)$ & 259 & $88(33,98 \%)$ & $128(49,42 \%)$ \\
\hline & $\begin{array}{l}\text { 24. Mejoran las infraestructuras básicas } \\
\text { (saneamiento, agua, teléfono, internet...) }\end{array}$ & 212 & $74(34,91 \%)$ & $101(47,64 \%)$ & 254 & $147(57,87 \%)$ & $48(18,90 \%)$ \\
\hline & $\begin{array}{l}25 \text {. Genera un problema de gestión de } \\
\text { residuos }\end{array}$ & 212 & $159(75,00 \%)$ & $31(14,62 \%)$ & 258 & $138(53,49 \%)$ & $61(23,64 \%)$ \\
\hline & $\begin{array}{l}\text { 26. Aumenta la contaminación (basura, } \\
\text { huidos...) }\end{array}$ & 212 & $115(54,25 \%)$ & $71(33,49 \%)$ & 260 & $141(54,23 \%)$ & $82(31,54 \%)$ \\
\hline
\end{tabular}

En la dimensión sociocultural (cuestiones 12-21), la percepción de efectos positivos no es tan mayoritaria como para los efectos económicos. La mayoría de los encuestados no perciben que la dotación y el acceso a los servicios, sanitarios y de transporte, se vean afectados ni positiva ni negativamente por la afluencia de peregrinos; ni tampoco que contribuya a incrementar la oferta de actividades culturales o de ocio. Tampoco perciben, mayoritariamente, que la existencia del Camino contribuya a mejorar la calidad del 
servicio en tiendas, restaurantes, etc. Sin embargo, en el ámbito puramente cultural, la percepción es de un impacto positivo. Mayoritariamente, los encuestados consideran que el Camino contribuye a mantener el patrimonio cultural (especialmente en Pedrafita) y, sobre todo, que es una oportunidad de intercambio cultural. En cuanto al coste social, en términos de mayores conflictos, en ambos municipios, la inmensa mayoría no perciben que el Camino genere conflictividad. En cuanto al efecto diferencial que pueda derivarse del hecho de que los peregrinos viajen solos o en pareja frente a hacerlo en grupos grandes, los encuestados no tienen una percepción clara.

En relación al impacto medioambiental (cuestiones 22-26), la percepción general en ambos municipios es que el Camino tiene claros efectos positivos. Mayoritariamente (64\%), los encuestados están de acuerdo en que el Camino contribuye a preservar el paisaje y, algo menos, consideran que favorece al cuidado de los núcleos de población. La percepción respecto a la contribución del Camino a la mejora en las infraestructuras básicas (saneamiento, agua, teléfono...) es diferente en los dos municipios analizados. Mientras en Pedrafita un $48 \%$ de los encuestados están de acuerdo, en Melide lo están únicamente un 19\%, estando en desacuerdo el 58\%. Estas percepciones están en línea con las mostradas en el ámbito sociocultural respecto a la provisión y acceso a servicios. En lo que coinciden los dos municipios es en no percibir efectos negativos, ni en términos de que se genere un problema en la gestión de residuos ni en que aumente la contaminación.

En la última parte del primer bloque del cuestionario se pretende obtener una valoración global del encuestado sobre la percepción del impacto del Camino, su actitud respecto a los peregrinos y la percepción de saturación (Figura 2). La primera cuestión, la percepción sobre el impacto demográfico del Camino, hace referencia a un aspecto muy relevante para la sostenibilidad futura de ambos municipios dado que se trata de territorios envejecidos y en regresión demográfica, por ello se incluyó de forma individual. La percepción sobre el impacto demográfico, en el sentido de que ayuda a mantener o incrementar la población residente, es más positiva en Pedrafita que en Melide, resultado que es consistente con el tamaño y la dinámica demográfica de cada municipio. En ambos casos, al ser preguntados, los residentes remarcan que la existencia del Camino ayuda a fijar población, pero no a su aumento. Es decir, son conscientes de que el municipio pierde población, pero la percepción es que la pérdida sería mayor en ausencia del Camino. ${ }^{8}$

8 Puesto que la tasa de crecimiento anual acumulativa fue del -2,5\% desde el inicio del siglo XXI, sin duda, los residentes entienden que el Camino tiene un efecto positivo sobre la dinámica demográfica comparándose con otras localidades semejantes por las que no discurre el Camino y cuya evolución ha sido mucho más negativa. 


\section{Figura 2 \\ PERCEPCIÓN DE IMPACTO Y SATURACIÓN DE LOS RESIDENTES Y ACTITUD HACIA EL CAMINO}



Fuente: Elaboración propia.

En cuanto a la percepción de saturación, si bien en ambos casos la respuesta mayoritaria es que están de acuerdo en que no hay demasiados peregrinos, ni siquiera algunos meses al año, hay ciertos matices. Mientras en Pedrafita no se percibe ningún tipo de saturación, en Melide, casi un tercio de los encuestados están de acuerdo en que, algunos meses al año, hay demasiados peregrinos; resultado que es coherente con el mayor número de peregrinos en Melide y con la mayor intensidad del contacto.

En los dos municipios analizados, la actitud ante el Camino es muy favorable, más del $75 \%$ de los encuestados están de acuerdo en que "personalmente, les agrada la presencia de peregrinos" y tan solo un $4 \%$ en Melide y un $6 \%$ en Pedrafita no están de acuerdo. La actitud tan favorable ante el Camino y la afluencia de peregrinos es consistente con los resultados alcanzados en el análisis de los distintos ámbitos de impactos percibidos.

Finalmente, cuando los residentes son preguntados por el impacto global, teniendo en cuenta todos los efectos para las distintas dimensiones, la percepción mayoritaria en ambos municipios, pero especialmente en Pedrafita, es que los beneficios son mayores que los costes y que, en los últimos años, el municipio prosperó como consecuencia del Camino. ${ }^{9}$

Para recoger información sobre la actitud de los residentes hacia el Camino de una forma alternativa, en el segundo bloque del cuestionario se les pidió que identificasen los que, a su juicio, son los tres principales problemas del municipio, entre los que se incluyó "el Camino y la afluencia de peregrinos". Los principales problemas señalados por los

9 Sin embargo, en línea con lo que ocurre con el impacto económico positivo, la percepción es mucho más positiva para los efectos a nivel del municipio que a nivel de la propia familia. Parece que los encuestados no perciben que la prosperidad del municipio se traduzca en una mejora de su vida cotidiana. 
residentes de ambos municipios son de tipo demográfico y económico (Figura 3), aunque con algunas diferencias derivadas de sus características.

\section{Figura 3 \\ PRINCIPALES PROBLEMAS DEL MUNICIPIO PERCIBIDOS POR LOS RESIDENTES}

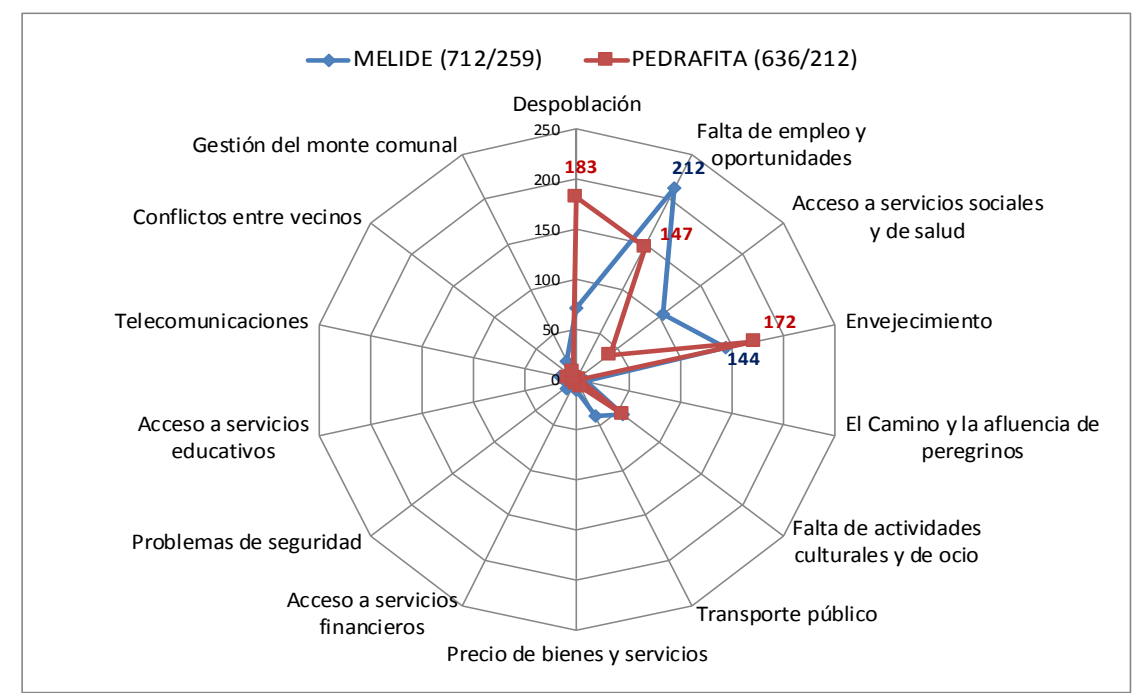

Fuente: Elaboración propia.

En el caso de Pedrafita, los principales problemas identificados son el envejecimiento de la población y la despoblación, seguidos de la falta de empleo y oportunidades. En Melide, la falta de empleo es percibida como el principal problema del municipio, seguido del envejecimiento demográfico. En este caso, en tercer lugar, se indica el acceso a servicios sociales y de salud. Resulta llamativo que en una cabecera comarcal con una mayor dotación de servicios este sea un problema de mayor calado que para los residentes en Pedrafita do Cebreiro. Pero debemos señalar que durante los meses de realización de la encuesta se produjo un conflicto sobre el retraso en la ejecución del nuevo centro de salud en Melide, lo que sin duda tuvo influencia en la percepción de los residentes sobre los problemas más relevantes de su municipio. En ningún caso, el Camino y la afluencia de peregrinos son vistos como un problema.

\subsection{Potenciales factores que influyen en la percepción. Análisis de independencia}

Tal como se mostró en el apartado 2, en la literatura se identifican varios factores que potencialmente influyen en la percepción que tienen los residentes del impacto del turismo en las distintas dimensiones. Algunos factores se corresponden con características sociodemográficas, mientras que otros tienen que ver con la relación de los residentes con el 
sector turístico y con los turistas; en particular, con la intensidad del contacto, la distancia al recurso turístico y la dependencia económica del turismo. En nuestro caso, analizamos si existen diferencias en la percepción del impacto que tienen los residentes en función de sus características, segmentando la muestra total según: (1) grupos de edad (18-39; 40-64;>65), (2) género (hombres; mujeres), (3) nivel de estudios (primarios; secundarios; superiores), (4) localización de la parroquia de residencia (en el Camino; fuera del Camino); (5) distancia reportada de la residencia al camino (<10minutos; entre 10 y 30 minutos; más de 30minutos); (6) frecuencia del contacto (ninguno; pocas veces; algunas veces; con frecuencia); (7) motivo del contacto (trabajo; resto de motivos) y (8) ingresos familiares vinculados al camino. ${ }^{10}$ Segmentada la muestra según estas características, se realizan contrastes de independencia.

Para la percepción de impacto global, impacto demográfico, saturación y la actitud ante el Camino (que se corresponden con la variable dependiente, que presenta tres categorías de nivel de acuerdo), la dependencia o independencia de la percepción respecto de cada una de las ocho variables independientes se analiza en función de los resultados de los contrastes Chi-cuadrado, $x^{2},{ }^{11}$ que se muestran en la Tabla 3.

La percepción de impacto global es, en general, independiente de las características de los residentes. Por lo tanto, los resultados de la valoración global de los impactos del Camino sobre la población local son positivos y muy robustos. Factores como el motivo del contacto con los peregrinos y la localización de la parroquia en relación al Camino, al igual que la distancia, no influyen en la percepción de los residentes. En cuanto al género, en el caso de Melide, los hombres tienen una percepción más favorable tanto del impacto global como del impacto demográfico; mientras que para el resto de los ítems la percepción es igual para hombres y mujeres, lo mismo que ocurre en Pedrafita para todos los ítems considerados. Respecto del nivel de estudios, en Pedrafita son los más educados los que tienen una mayor percepción de impacto demográfico, ya que seguramente entienden mejor que, a pesar de perder población el municipio, el efecto fue positivo, como se comentó anteriormente; también son los más educados los que tienen mayor percepción de saturación.

La percepción de impacto del Camino es diferente en función de la edad del residente. En ambos municipios, las personas mayores valoran más que los jóvenes todos los impactos relacionados con el Camino y tienen una actitud más favorable. Este resultado, si bien está en línea con lo alcanzado en otros trabajos (Tomljenovic y Faulkner, 2000; McGehee y Andereck, 2004), no es el generalmente observado en la literatura. En la mayor parte de los estudios sobre percepción de impacto del turismo, la edad tiene efectos mixtos (Harrill,

10 Consideramos que los ingresos de la unidad familiar del residente están "vinculados al Camino" cuando dice estar de acuerdo (bastante o muy) con el ítem "La existencia del Camino contribuye a la mejora de los ingresos y el nivel de vida de la familia", mientras que si dice estar en desacuerdo (bastante o muy) consideramos que "no están vinculados".

11 Se calcula el estadístico Chi-cuadrado, siendo la hipótesis nula que las variables son independientes (la percepción no se ve afectada por el segmento concreto) y la hipótesis alternativa que las variables están relacionadas, es decir, que la variable dependiente (la percepción de impacto) sí se ve afectada o es estadísticamente diferente para los distintos segmentos $\chi^{2}=\sum_{i=1}^{k} \frac{\left(O_{i}-E_{i}\right)^{2}}{E_{i}}$, donde $O_{i}$ son las frecuencias observadas o empíricas y $E_{i}$ las esperadas o teóricas. 




Cuadernos de Turismo, 47, (2021), 37-63 


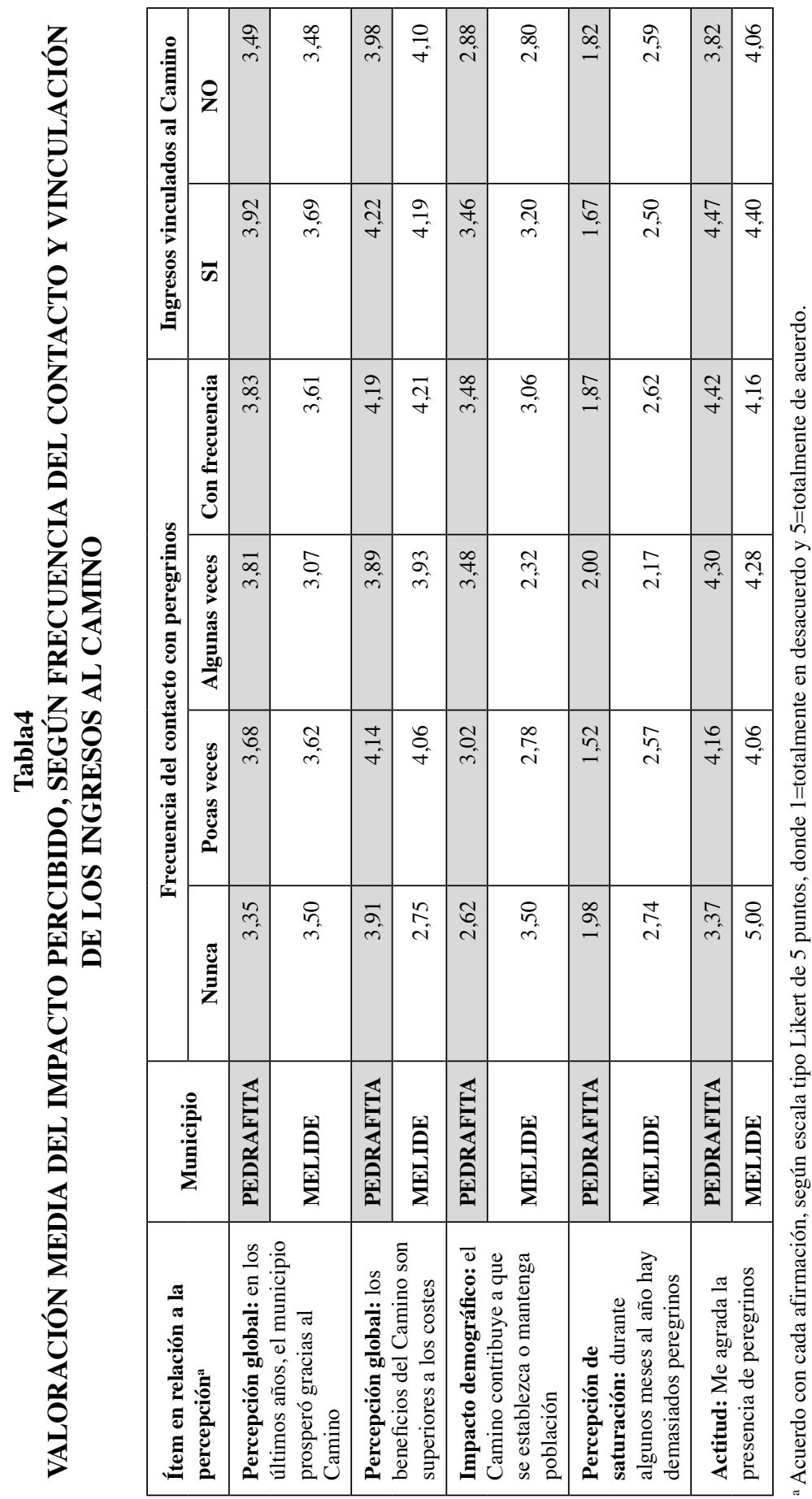


2004) o no tiene ningún efecto (Snaithy Haley, 1999) o lo tiene en el sentido contrario al observado en estos municipios (Cavus y Tanrisevdi, 2002); lo cual, siguiendo la teoría del intercambio social (Ap, 1992), estaría justificado porque los mayores no pueden compensar personalmente los impactos negativos con las potenciales ganancias. Profundizar en esta relación es importante para monitorizar la evolución futura de la percepción en ambas localidades.

Las principales diferencias de percepción observadas, entre los dos municipios y entre los diferentes ítems, se relacionan con la frecuencia del contacto y con la dependencia de los ingresos de los residentes respecto de actividades vinculadas al Camino. El valor medio de las percepciones que tienen los distintos grupos para los ítems considerados se muestra en la Tabla 4. Aunque la frecuencia del contacto estaría, en principio, relacionada con la distancia, los dos conceptos no son sinónimos, ya que los residentes pueden estar en contacto con los visitantes en muchas situaciones diferentes (Deery et al., 2012). Así, aunque la distancia no parece tener ninguna influencia, la frecuencia del contacto con los peregrinos influye en la actitud y la percepción de saturación en el caso de Pedrafita. Cuanto mayor es el contacto, más favorable es la actitud y menor es la percepción de saturación, resultado que está en línea con lo sugerido en parte de la literatura (Andereck et al., 2007; Andereck et al., 2005; Akis et al., 1996; Lankford y Howard, 1994), que concluye que cuanto mayor es el conocimiento que los residentes tienen del turismo y mayor es el contacto con los turistas, más positiva es la percepción que tienen de las ganancias del turismo y, por tanto, más favorable la actitud. ${ }^{12}$ En cuanto a la dependencia de los ingresos, los residentes con ingresos vinculados a la existencia del Camino tienen una percepción más positiva del impacto global y del impacto demográfico, así como una actitud más favorable ante la presencia de peregrinos y una menor percepción de saturación, resultado ampliamente reconocido, de manera no ambigua, en la literatura (Andereck et al., 2007; Andereck et al., 2005; Andriotis, 2005; Haley et al., 2005; Akis et al., 1996; Jonhson et al., 1994; Perdue et al., 1990).

\section{CONCLUSIONES}

Desde una perspectiva general, los resultados del estudio muestran que los residentes de los dos municipios analizados consideran que la existencia del Camino de Santiago y la afluencia de peregrinos generan más beneficios que perjuicios, y que ha contribuido a la prosperidad de estos municipios; resultados que, en general, son independientes de las características de los residentes, si bien tienden a ser más positivos para aquellos cuyos ingresos están vinculados al Camino. Mayoritariamente, los residentes de ambos municipios perciben un impacto económico positivo (para ingresos, comercio local, empleo, turismo, otras actividades no turísticas) y no perciben que se genere un impacto negativo, en cuanto a que perjudique el desarrollo de otras actividades económicas ni contribuya a un incremento de los precios, salvo en la hostelería. Además, en ambos municipios, se considera que los beneficios generados son para los residentes del municipio, si bien esos beneficios repercuten

12 En este caso, los resultados para Melide se ven afectados por el hecho de que muy pocos residentes entrevistados (sólo 4) no tienen ningún contacto con los peregrinos. 
en unos pocos residentes; percepción más extendida en Pedrafita, donde, mayoritariamente, se percibe que el Camino es la principal activad económica del municipio.

En cuanto al impacto social, la percepción mayoritaria es que la provisión y el acceso a servicios (sanitarios, sociales, transporte) no se ven afectados, ni positiva ni negativamente, por el Camino. Mayoritariamente, los residentes tampoco perciben que el Camino contribuya a aumentar la oferta de actividades culturales o de ocio. Sin embargo, hay una percepción mayoritaria de un impacto cultural positivo, sobre todo en relación con la contribución del Camino a la conservación del patrimonio cultural y a las oportunidades de intercambio cultural que ofrece. En ningún caso, los residentes perciben que el Camino contribuya a incrementar la conflictividad en sus comunidades. La percepción de impacto demográfico es más positiva en Pedrafita que en Melide, consistente con el tamaño, la dinámica demográfica y la valoración relativa del Camino en cada municipio, y, en mayor medida, por parte de los residentes con mayor nivel de estudios y los que tienen ingresos derivados de actividades vinculadas al Camino. Los residentes de ambos municipios perciben un impacto medioambiental positivo. Mayoritariamente, no perciben efectos negativos (para la gestión de residuos o los niveles de contaminación) y sí perciben una contribución positiva, más para el cuidado del paisaje que para el cuidado de los núcleos de población.

Después de la valoración conjunta, se analizaron los resultados segmentando la muestra por edad, género, nivel de estudios, distancia de la residencia al Camino, intensidad del contacto con los peregrinos y vinculación de los ingresos familiares a actividades relacionadas con el Camino. Aunque con ciertas matizaciones, en general, se mantiene para los diferentes grupos la percepción de un impacto positivo y de ausencia de saturación, así como la actitud favorable ante el Camino y la presencia de peregrinos. La edad influye en la percepción de saturación (menor para los mayores) y en la actitud (más favorable para los mayores), mientras que la localización de la residencia habitual o la distancia no parecen influir significativamente en las valoraciones. Las mayores diferencias se producen en relación a la frecuencia del contacto, (cuanto mayor es el contacto, más favorable es la actitud y menor es la percepción de saturación), y la dependencia de los ingresos, (los residentes con ingresos vinculados a la existencia del Camino tienen una percepción más positiva del impacto global y del impacto demográfico, así como una actitud más favorable ante la presencia de peregrinos y una menor percepción de saturación).

A la vista de los impactos positivos percibidos del Camino, en particular en los ámbitos económico (actividad y empleo) y demográfico, y de los problemas de los municipios identificados, en esos mismos ámbitos, podríamos concluir que los residentes consideran la existencia del Camino de Santiago como parte de la solución a los problemas del municipio, especialmente en el caso de Pedrafita de Cebreiro (el municipio más rural, pequeño y envejecido). Además, muy mayoritariamente, los residentes no perciben que exista saturación del Camino, ni siquiera en los meses de mayor afluencia de peregrinos. Todo ello explicaría la actitud tan favorable hacia el Camino y la presencia de peregrinos que muestran los resultados en ambos municipios. 


\section{REFERENCIAS}

ABDOLLAHZADEH, G. y SHARIFZADEF, A. (2014): "Rural Residents' Perceptions Toward Tourism Development: a Study from Iran", International Journal of Tourism Research, vol. 16 (2), pp. 126-136.

AGUILÓ, E. y ROSSELLÓ, J. (2005): «Host Community Perceptions: A Cluster Analysis», Annals of Tourism Research, vol. 32 (4), pp. 925-941.

AKIS, S., PERISTIANIS, N. y WARNER, J. (1996): «Residents' attitudes to tourism development: the case of Cyprus», Tourism Management, vol. 17 (7), pp.481-404.

ALLEN, L., LONG, P. PERDUE, R. y KIESELBACH, S. (1988): «The Impact of Tourism Development on Residents' Perceptions of Com- munity Life», Journal of Travel Research, vol. 27 (1), pp. 16-21.

ALLEN, L., HAFER, H., LONG, P. y PERDUE, R. (1993): «Rural residents' attitudes toward recreation and tourism development», Journal of Travel Research, vol. 31 (4), pp. 27-33.

ALMEIDA-GARCÍA, F., PELAEZ-FERNANDEZ, M., CORTES-MACIAS, A. y BALBUENA-VAZQUES, R. (2016): "Residents' perceptions of tourism development in Benalmádena (Spain)", Tourism Management, vol. 54, pp. 259-274.

ANDERECK, K., VALENTINE, K. KNOPF, R. y VOGT, C. (2005): «Residents' perceptions of community tourism impacts», Annals of Tourism Research, vol. 32 (4), pp. 1.056-1.076.

ANDERECK, K., VALENTINE, K., VOGT, C. y KNOPF, R. (2007): «A cross-cultural analysis of tourism and quality of live perceptions», Journal of Sustainable Tourism, vol. 15 (5), pp. 483-502.

ANDRADE-SUÁREZ, M. J. (2011): «Consideraciones generales sobre la potencialidad del turismo para el desenvolvimiento rural: El Camino de Santiago como estudio de caso», TurYDes Revista de Investigación en Turismo y desarrollo local, vol. 4 (9), pp. 1-32.

ANDRADE, M. J., LEIRA, G. y CARAMÉS, R. (2010): «El turismo como factor estratégico de desarrollo rural: El Camino de Santiago Francés», ROTUR/ Revista de Ocio y Turismo, $\mathrm{n}^{\circ} 3$, pp. 49-82.

ANDRIOTIS, K. (2005): «Community groups' perceptions of and preferences for tourism development: evidence from Crete», Journal of Hospitality and Tourism Research, vol. 29, pp. 67-90.

ANDRIOTIS, K. y VAUGHN, D. (2003): «Urban residents' attitudes towards tourism development: the case of Crete», Journal of Travel Research, vol. 42 (2), pp.172-185.

AP, J. (1990): «Residents' Perceptions Research on the Social Impacts of Tourism», Annals of Tourism Research, vol. 17, pp. 610-616.

AP, J. (1992): «Resident's perceptions on tourism impacts», Annals of Tourism Research, vol. 19 (4), pp. 665-690.

BELISLE, F. y HOY, D. (1980): «The Perceived Impact of Tourism by Residents: A Case Study in Santa Marta, Columbia», Annals of Tourism Research, vol. 7, pp. 83-101.

BESCULIDES, A., LEE, M. y MCCORMICK, P. (2002): «Residents' perceptions of the cultural benefits of tourism», Annals of Tourism Research, vol. 29 (8), pp. 303-319. 
BRIDA, J., DISEGNA, M. y OSTI, L. (2014): «Resident' Perceptions of Tourism Impacts and Attitudes towards Tourism Policies», TOURISMOS: An International Multidisciplinary Journal of Tourism, vol. 9 (1), pp. 37-71.

BRUNT, P. y COURTNEY, P. (1999): «Host perceptions of sociocultural impacts», Annals of Tourism Research, vol. 26 (3), pp. 493-515.

CASTILLO, A., SOTO, M. y LÓPEZ, T. (2012): «Percepción y Actitudes del Residente acerca del Impacto del Turismo en la Isla de Santiago (Cabo Verde) », TurYDes Revista de Investigación en Turismo y Desarrollo Local, vol. 5 (12), pp. 1-23.

CAVUS, S. y TANRISEVDI, A. (2002): «Residents' attitudes toward tourism development: A case study in Kusadasi, Turkey», Tourism Analysis, vol. 7 (3/4), pp. 259-269.

CHEN, S. (2011): "Residents' Perceptions of the Impact of Major Annual Tourism Events in Macao: Cluster Analysis", Journal of Convention \& Event Tourism, vol. 12, pp.106-128.

CHOI, C. y MURRAY, I. (2010): «Resident attitudes towards sustainable community tourism», Journal of Sustainable Tourism, vol. 18 (4), pp. 575-594.

DAVIS, D., ALLEN, J. y COSENZA, R. (1988): «Segmenting local residents by their attitudes, interests, and opinions toward tourism», Journal of Travel Research, vol. 27, pp. 2-8.

DEERY, M., JAGO, L. y FREDLINE, L. (2012): «Rethinking social impacts of tourism research: a new research agenda», Tourism Management, vol. 33, pp. 64-73.

DÍAZ, R. y GUITIÉRREZ, D. (2010): «La actitud del residente en el destino turístico de Tenerife: evaluación y tendencia», PASOS, Revista de Turismo y Patrimonio Cultural, vol. 8 (4), pp.431-444.

EASTERLING, D. (2004): «The residents' perspective in tourism research: a review and synthesis», Journal of Travel \& Tourism Marketing, vol. 17, pp. 45-62.

FRAUMAN, E. y BANKS, S. (2011): «Gateway community resident perceptions of tourism development: Incorporating importance-performance analysis into the limits of acceptable change framework», Tourism Management, vol. 13 (1), pp. 128-140.

GARROD, F. y FYALL, A. (1998): «Beyond the rhetoric of sustainable tourism?», Tourism Management, vol. 19 (3), pp.199-212.

GIGIREY, A. (2003): «Turismo cultural en lugares sagrados desde la perspectiva de los residentes. Un estudio de la catedral de Santiago de Compostela», Estudios Turísticos, $\mathrm{n}^{\circ} 158$, pp. 79-108.

GURSOY, D., JUROWSKI, C. y UYSAL, M. (2002): «Resident attitudes: A structural modeling approach», Annals of Tourism Research, vol. 29, pp. 79-105.

GURSOY, D. y RUTHERFORD, D. (2004): «Host Attitudes toward Tourism: An Improved Structural Model», Annals of Tourism Research, vol. 31 (3), pp. 495-516.

GURSOY, D., CHI, C. y DYER, P. (2009): «An Examination of Locals’ Attitudes», Annals of Tourism Research», vol. 36 (4), pp. 723-726.

HALEY, A.J., SNAITH, T. y MILLER, G. (2005): «The social impacts of tourism: a case study of Bath», UK. Annals of Tourism Research, 32 (3), pp. 647- 668.

HARRILL, R. (2004): «Residents' Attitudes toward Tourism Development: a Literature Review with Implications for Tourism Planning», Journal of Planning Literature, vol. 18 (3), pp. 251-266. 
HARALAMBOPOULOS, N. y PIZAM, A. (1996): «Perceived impacts of tourism: The case of Samos», Annals of Tourism Research, vol. 23 (3), pp. 503-526.

HILLERY, M., NANCARROW, B. GRIFFIN, G. y SYME, G. (2001): «Tourist Perception of Environmental Impact», Annals of Tourism Research, vol. 28 (4), pp. 853-867.

JOHNSON, J., SNEPENGER, D. y AKIS, S. (1994): «Residents' Perceptions of Tourism Development», Annals of Tourism Research, vol. 21, pp. 629-642.

JUROWSKI, C., UYSAL, M. y WILLIAMS, R. (1997): «A Theoretical Analysis of Host community Resident Reactions to Tourism», Journal of Travel Research,36 (2), pp.3-11.

JUROWSKI, C. y GURSOY, D. (2004): «Distance effects on residents' attitudes toward tourism», Annals of Tourism Research, vol. 31 (2), pp. 296-312.

KEOGH, B. (1990): «Resident Recreationists' Perceptions and Attitudes with Respect to Tourism Development», Journal of Applied Recreation Research, vol. 15 (2), pp. 71-83.

KIM, K., UYSAL, M. y SIRGY, M. (2013): «How does tourism in a community impact the quality of life of community residents?», Tourism Management, vol. 36, pp. 527-540.

KREAG, G. (2001): The Impacts of Tourism. Minnesota, University of Minnesota Press,

LANKFORD, S. (1994): «Attitudes and perceptions toward tourism and rural regional development», Journal of Travel Research, vol. 32 (3), pp.35-33.

LANKFORD, S. y HOWARD, D. (1994): «Developing a Tourism Impact Attitude Scale», Annals of Tourism Research, vol. 21, pp. 121-139.

LEE, T. H. (2013). «Influence analysis of community resident support for sustainable tourism development», Tourism Management, vol. 34, pp. 37-46.

LIU, J. C., y VAR, T. (1986): «Resident attitudes toward tourism impacts in Hawaii», Annals of Tourism Research, vol. 13, pp. 193-214.

LOIS-GONZÁLEZ, R., SANTOS, X. y TABOADA, R. (2018): «The Camino de Santiago de Compostela: The Most Important Historic Pilgrimage Way in Europe», en Olsen, D. y Trono, A. (Eds.): Religious pilgrimage routes and trails: sustainable development and management, CAB International, UK, pp.72-87.

LONG, P. T., R. R. PERDUE, y L. ALLEN (1990): «Rural Resident Tourism Perceptions and Attitudes by Community Level of Tourism», Journal of Travel Research, vol. 28 (3), pp. 3-9.

MAAK, K. (2009): «El Camino de Santiago como posible motor turístico en zonas rurales de escasos recursos: el caso de Brandeburgo», Cuadernos de Turismo, $\mathrm{n}^{\circ} 23$, pp. 149- 171.

MADRIGAL, R. (1993): «A tale of tourism in two cities», Annals of Tourism Research, vol. 20, pp. 336-353.

MARTÍN-DUQUE, C. (2014): «El Camino de Santiago Primitivo a su paso por Asturias, estudio de una ruta cultural desde la perspectiva turística», Cuadernos de Turismo, $\mathrm{n}^{\circ}$ 33, pp. 233-249.

MARTÍN-DUQUE, C. (2017): «Los impactos del turismo en el Camino de Santiago Francés: una aproximación cualitativa», Methaodos. Revista de ciencias sociales, vol. 5 (1), pp. 62-73. 
MATHIESON, A. y WALL, G. (1982): Tourism: Economic, Physical, and Social Impacts. New York: Longman House.

MCGEHEE, N. y ANDERECK, K. (2004): «Factors predicting rural residents' support of tourism», Journal of Travel Research, vol. 43, pp. 131-140.

MULER GONZÁLEZ, V., COROMINA, L. y GALI, N. (2018): “Overtourism: residents' perceptions of tourism impact as an indicator of resident social carrying capacity-case study of a Spanish heritage town", Tourism Review, vol. 73 (3), pp. 277-296.

MURPHY, P. E. (1981): «Community Attitudes to Tourism», Tourism Management, vol. 2 (3), pp. 189-195.

MURPHY, P. E. (1985): Tourism: A Community Approach. New York, Routledge.

NICHOLAS, L., THAPA, B. y KO, Y. (2009): «Resident' perspectives of A World Heritage Site: The Pitons Management Area, St. Lucia», Annals of Tourism Research, vol. 36 (3), pp. 390-412.

PARDELLAS, X. y PADÍN, C. (2014): «El Camino de Santiago Portugués en Galicia y las estrategias turísticas locales», Revista de Análisis Turístico, 17, pp.51-59.

PERDUE, R., LONG, P. y ALLEN, L. (1990): «Resident Support for Tourism Development»,Annals of Tourism Research, vol. 17 (4), pp. 586-599.

PIZAM, A. (1978): «Tourist Impacts. The Social Costs to the Destination Community as Perceived by its Residents», Journal of Travel Research, vol. 16, pp.8-12.

PORCAL, M., DÍEZ, A. y JUNGUITO, J. (2012): «Dimensión territorial y turística de la ruta Norte del Camino de Santiago en el País Vasco: Distintas concepciones, valoraciones y propuestas de intervención sobre un fenómeno multifacético», Boletín de la Asociación de Geógrafos Españoles, $\mathrm{n}^{\circ}$ 58, pp. 177-204.

PRECEDO, A., REVILLA, A. y MÍGUEZ, A. (2007): «El turismo cultural como factor estratégico de desarrollo: el Camino de Santiago», Estudios Geográficos, vol. 68 (262), pp. 205-234.

RASOOLIMANESH, M., JAAFAR, M., KOCK, N. y RAMAYAH, N. (2015): “A revised framework of social exchange theory to investigate the factors influencing residents' perceptions“, Tourism Management Perspectives, vol. 16, pp. 335-345.

SANTOS SOLLA, J.M. y LÓPEZ, L. (2015): «Tourism Policies in A WHC: Santiago de Compostela (Spain) », International Journal of Research in Tourism and Hospitality, vol. 1 (2), pp. 1-8.

SHARIFF, N. y TAHIR, S. (2003): «Residents' Attitudes toward Impacts of Tourism: A Case Study of Langkawi, Malaysia», Malaysian Management Journal, vol. 7 (2), pp. 13-24.

SHARMA, B. y DYER, P. (2009): «An investigation of differences in residents' perceptions on the Sunshine Coast: Tourism impacts and demographic variables», Tourism Geographies, vol. 11 (2), pp.187-213.

SHARPLEY, R. (2014): «Host perceptions of tourism: A review of the research», Tourism Management, vol. 42, pp. 37-49.

SHELDON, P. y ABENOJA, T. (2001): «Resident Attitudes in a Mature Destination: the Case of Waikiki», Tourism Management, vol. 22 (5), pp. 435-443.

SHELDON, P. y VAR, T. (1984): «Residents attitudes toward tourism in North Wales», Tourism Management, 5 (1), pp.40-47. 
SNAITH, T. y HALEY, A. (1999): «Residents' opinions of tourism development in the historic city of York, England», Tourism Management, vol. 20 (5), pp. 595-603.

TOMLJENOVIC, R., y FAULKNER, B. (2000): «Tourism and older residents in a Sunbelt resort», Annals of Tourism Research, vol. 27 (1), pp. 93-114.

TOSUN, C. (2002): «Host Perceptions of Impacts: A Comparative Tourism Study», Annals of Tourism Research, vol. 29 (1), pp.231-253.

TOVAR, C. y LOCKWOOD, M. (2008): «Social impacts of tourism: an Australian regional case study», International Journal of Tourism Research, vol. 10 (4), pp. 365378.

TYRRELL, T. y SPAULDING, P. (1984): «A Survey of Attitudes toward Tourism Growth in Rhode Island», Hospitality Education and Research Journal, vol. 8, pp. 22-23.

VARGAS-SÁNCHEZ, A., DE LOS ÁNGELES PLAZA-MEJÍA, M. y PORRAS-BUENO, N. (2009): «Understanding residents' attitudes toward the development of industrial tourism in a former mining», Journal of Travel Research, vol. 47 (3), pp. 373-387.

VARGAS-SÁNCHEZ, A., PORRAS-BUENO, N. y DE LOS ÁNGELES PLAZA-MEJÍA, M. (2011): «Explaining residents' attitudes to tourism. Is a universal model possible?», Annals of Tourism Research, vol. 38 (2), pp. 460-480.

WALL, G. y MATHIESON, A. (2006): Tourism: Changes, Impacts, and Opportunities. Nueva York, Pearson Education, Upper Saddle River.

WILLIAMS, J. y LAWSON, R. (2001): "Community issues and resident opinions of tourism», Annals of Tourism Research, vol. 28 (2), pp. 269-290. 